\title{
1 Regionalization, constraints, and the ancestral ossification 2 patterns in the vertebral column of amniotes
}

4 Antoine Verrière ${ }^{1 *}$, Nadia B. Fröbisch ${ }^{1}$, Jörg Fröbisch ${ }^{1,2}$

$5{ }^{1}$ Museum für Naturkunde, Leibniz-Institut für Evolutions- und Biodiversitätsforschung,

6 Invalidenstraße 43, 10115 Berlin, Germany

72 Institut für Biologie, Humboldt-Universität zu Berlin, Unter den Linden 6, 10099 Berlin,

8 Germany

10 Keywords

11 Vertebral column; ossification; neurocentral fusion; development; amniotes.

\section{Author Contributions}

14 AV conducted the analysis, did the bibliographic research work, and made the figures.

$15 \mathrm{AV}, \mathrm{JF}$, and NF wrote the manuscript.

\section{Abstract}

The development of the vertebral column has been studied extensively in modern amniotes, yet many aspects of its evolutionary history remain enigmatic. Here we expand the existing data on four major vertebral developmental patterns in amniotes based on exceptionally well-preserved specimens of the early Permian mesosaurid reptile

22 Stereosternum: (i) centrum ossification, (ii) neural arch ossification, (iii) neural arch fusion,

23 and (iv) neurocentral fusion. We retrace the evolutionary history of each pattern and

24 reconstruct the ancestral condition in amniotes. Despite 300 million years of evolutionary 25 history, vertebral development patterns show a surprisingly stability in amniotes since their 26 common ancestor. We propose that this conservatism may be linked to constraints posed 27 by underlying developmental processes across amniotes. However, we also point out that 28 mammals and birds differ more strongly from the ancestral condition than other clades, 29 which might be linked to a stronger regionalization of the column in these two clades.

\section{Introduction}


The vertebral column is the defining feature of all vertebrates and has long been the subject of special attention by developmental and evolutionary biologists, yet certain key aspects of its development and evolution remain unexplored. Thanks to a large body of research spanning more than a century (Gadow, 1896; Kölliker, 1876; Lawson and Harfe, 2017), the way vertebrae are formed throughout ontogeny is generally well understood. Vertebrae ossify via endochondral ossification, meaning that embryonic cartilaginous frameworks progressively mineralize into fully ossified elements. Ossification starts at one or several centers of ossification inside the cartilage matrix and spreads from there until the matrix is entirely replaced by bone.

In amniotes, elements of the vertebral column undergo two stages of endochondral ossification. The first stage, referred to as primary ossification, consists of the mineralization of the neural arch and pleurocentrum. As the amphibian intercentrum is lost in amniotes (Clack, 2012), we only consider the pleurocentrum here and refer to it as centrum for the purpose of this study. The paired elements of the neural arch arise from two ossification centers, one on each side, and eventually fuse dorsomedially. The mineralization of the centrum starts in a pair of ventrally located ossification centers (Danto et al., 2017) and then spreads through the centrum. Eventually, centrum and neural arch come into contact forming what is called the neurocentral suture. It is only much later in development that this suture closes and both elements merge into a single vertebral unit in most amniotes. The second stage of vertebral ossification, or secondary ossification, is marked by the mineralization of the transverse processes and the spinous process.

Ossification events do not occur simultaneously throughout the vertebral column. On the contrary, they can originate in different locations along the vertebral column, occur at different times, and progress at different speeds and in different directions. In a given ossification pattern, it is generally possible to identify one or multiple spots from which ossification spreads, whether it is the first vertebrae where ossification centers are visible or the first vertebrae in which given elements begin to fuse. For clarity, we will indistinctly refer to these pattern starting points for initial ossification as loci (Fig. 1). Loci are not to

60 be confused with ossification centers, which are the points where ossification begins within 61 a single vertebra.

62 Although the sequence of ossification events leading to the formation of a single 63 vertebra in amniotes has been extensively studied (Böhmer, 2017; Bui and Larsson, 2021;

64 Zhang, 2009), the timing of occurrence and spatial progression of these events along the vertebral column has rarely been documented.. Even for model organisms, most studies 
66 only mention which of the neural arches or centra ossify first, if at all. With few exceptions

67 (Hautier et al., 2011, 2010), the disparity between patterns of vertebral development in 68 amniotes remains virtually unstudied, especially with respect to their evolutionary history.

69 While this is partly due to the infrequent preservation of ontogenetic series in the fossil 70 record, the axial column has also suffered from a limited research interest in 71 developmental paleontology. Neurocentral fusion (fusion of neural arches to the centra) 72 constitutes the only notable exception to this as it has been used as a proxy for maturity 73 in fossil archosaurs, especially in dinosaurs. Consequently, this fusion pattern is relatively 74 well documented for fossil members of this clade, albeit in a different context than in this 75 study (Irmis, 2007).

76 Vertebral elements are considered serially homologous within Amniota (Fleming et al., 77 2015), which allows for comparisons between clades. Here, we review the current state of 78 knowledge on the four major patterns of primary ossification of the vertebral column in 79 living and fossil amniotes (Fig. 1): (i) the ossification of pleurocentra (ii) the ossification of 80 neural arches, (iii) the fusion of paired neural arch elements, and (iv) neurocentral fusion. 81 These patterns are easily observed on the skeleton and therefore more likely to be 82 preserved in a fossil. In addition, thanks to some exceptionally well-preserved fossils of 83 the early Permian mesosaurid reptile Stereosternum tumidum, we provide the first 84 documentation of vertebral ossification patterns in an early amniote. We compiled data on 85 each of these patterns from the literature and used ancestral state reconstruction (ASR) 86 to trace their evolutionary history in the amniote clade. Finally, we reconstructed the 87 hypothetical ancestral condition for each of the patterns in amniotes.

88 Remarkably, we find that despite the 300-million-year history and great morphological 89 diversity, axial ossification patterns seem to be relatively conservative within Amniota. Our 90 results also show that the evolution of axial development was marked by the additional 91 acquisition and shifting of loci in several clades of amniotes, notably in mammals and 92 birds, which may be linked to the increased regionalization of the vertebral column in these 93 two clades.

\section{Results}

96 Centrum ossification

97 We were able to document the primary sequences of vertebral ossification in the fossil 98 mesosaurid reptile Stereosternum based on the juvenile specimen SMF-R-4512. In this 99 specimen, we could identify differences in the degree of ossification of some bones based 
100 on variations in color and robustness. SMF-R-4512 shows a stronger coloration and 101 thicker bone in the centra and neural arches of the cervical vertebrae as compared to other 102 regions of the vertebral column (Fig. 2A), indicating a more advanced ossification in this 103 region. This suggests that, in Stereosternum, centra and neural arches each had a single 104 anterior cervical locus of ossification and that ossification progressed posteriorly from 105 there.

106 The single anterior locus condition appears to be quite common among amniotes. Out 107 of the 41 documented taxa, 25 present a cervical locus of ossification for the centra (Table 108 S1). All birds and reptiles have a cervical locus, but most mammals do not. Our analysis 109 shows that the cervical locus has been lost in mammals, with this loss happening more or 110 less early in the history of mammals depending on the ASR model used: in Mammalia with 111 maximum likelihood, in Theria with parsimony.

112 Later, a few isolated mammalian taxa (Didelphis, Oryctolagus, Sus, and Tachyglossus) 113 reacquire the cervical locus (Table S1). With this exception, this locus is a phylogenetically 114 constrained trait and does not seem very plastic. Thus, rather than completely 115 disappearing, the genetic potential for a cervical locus might have been merely muted in 116 mammals and later reactivated in the aforementioned taxa. The potential causes of this 117 reactivation remain to be investigated, since there is no clear common denominator to 118 these taxa.

119 Centrum ossification is the only axial ossification pattern we reconstruct as having two 120 loci in amniotes ancestrally: one in the cervicals and one in the thoracics. Virtually all 121 mammals studied have a thoracic locus of central ossification; only Talpidae do not bear 122 the thoracic locus and have a sacral locus instead (Hautier et al., 2010). Some birds also 123 possess an additional locus of ossification in the upper dorsal region, and this locus is 124 reconstructed as ancestrally present in paleognathous birds (Fig. S1, S2). Didelphis and 125 Oryctolagus present an additional lumbar locus of ossification (Table S1). We measure a 126 very strong phylogenetic signal for each of the four loci positions $(\lambda=1.000, p<0.001)$.

127 The evolutionary scenario for the thoracic locus follows a reverse path to that of the 128 cervical locus: it is ancestrally present in amniotes, lost in reptiles but retained in 129 mammals. The thoracic locus appears to be already absent in Stereosternum, which would 130 suggest it may too have been muted or lost very early on in the history of Reptilia. After its 131 loss, the thoracic locus is reactivated or reacquired only twice in the reptilian lineage: in 132 paleognathous birds and in Sterna. While the peculiarity of development patterns in 
133 paleognaths has been pointed out (Maxwell and Larsson, 2009) and could be linked to the 134 reacquisition of the thoracic locus, its presence in Sterna remains enigmatic.

135 The moles Talpa and Mogera are the only mammals lacking a thoracic locus and 136 possessing a lumbar locus instead. In this case, the locus is located in the first lumbar, a 137 little more posteriorly than in other mammals, and could be the result of a slightly shifted 138 timing of ossification of the same overall area. However, the additional sacral locus found 139 in the two moles is clearly distinct and unique. Prochel (2006) identified that ossification 140 timing in Talpidae diverges significantly from the standard mammalian condition due to 141 their fossorial lifestyle, and it is very likely that a divergence in timing would also affect 142 axial ossification loci.

Neural arch ossification

145 Much like centrum ossification, neural arch ossification in the mesosaur Stereosternum 146 is progressing posteriorly from a single cervical locus (Fig. 2A). This cervical locus of 147 neural arch ossification is present in all amniote taxa (Fig. 3B). Although it is sometimes 148 found in association with other loci, the cervical locus is in fact the only locus that is present 149 in all reptiles, birds, marsupials, as well as in the temnospondyl Micromelerpeton (Table 150 S1).

Additional neural arch loci are only found within Mammalia. All placentals possess a second locus of neural arch ossification in the thoracic region, as does the monotreme Tachyglossus (Table S1). Notably, marsupials do not have any additional neural arch loci, 154 with the exception of Didelphis and Sminthopsis (Table S1). Bos, Capra, Dasypus, and 155 Homo also display a third neural arch locus in the lumbar region. The distribution of the 156 thoracic locus, which is restricted to mammals, shows a strong phylogenetic signal $(\lambda=$ 157 0.816, $p<0.001)$. Similarly, we find a significant phylogenetic signal in the distribution of 158 the lumbar locus $(\lambda=1.000, p=0.004)$.

159 Our ASR recovers the single cervical locus as ancestrally present in all clades of 160 amniotes and as the plesiomorphic condition in Amniota itself (Fig. 3B). The thoracic locus 161 is ancestral to all clades of mammals but is lost in Australidelphia. This locus is also absent 162 in all other non-mammalian amniotes (Fig. S3, S4). Likewise, the lumbar locus is 163 reconstructed as ancestral in bovids but as ancestrally absent in other super-generic 164 clades of amniotes. Parsimony mostly agrees with maximum likelihood and is only 165 inconclusive about the ancestral presence of a thoracic locus in two clades of Australian marsupials (Fig. S4). 
Neural arch fusion

Neural arch fusion is the least well documented of the four patterns we considered. To the best of our knowledge, it has only been described in 18 extant amniotes, and never in any fossil tetrapod. Here, we document the first occurrence of this process in the mesosaur Stereosternum. We made our observations on three specimens of Stereosternum: BSPG 1979 I 37, MZSP-PV 1301, and MZSP-PV 1313. All three of them have dorsally fused neural laminae in the cervical region whereas they are still unfused in posterior parts of the body. This suggests that neural arches fuse dorsally along an anteroposterior gradient, closing like a zipper. This "zipper-like" pattern is best observed in BSPG 1979 I 37 (Fig. 2B).

Unlike centrum and neural arch ossification, all studied taxa bear only one locus of neural arch fusion, which is located in the cervical or in the caudal section of the vertebral column (Fig. 3C). Interestingly, the cervical and caudal loci seem to be mutually exclusive since we found no taxon where both conditions overlapped. Moreover, the distribution of the neural arch fusion loci among amniotes is much less phylogenetically restricted than that of the ossification loci (Fig. 3C). Among the studied reptiles, three archosaurs, two squamates, and the mesosaur Stereosternum, possess a cervical locus, whereas only Bradypus, Homo, and Bos have this locus among mammals (Table S1). The caudal locus is found in all the studied marsupials, rodents, and lagomorphs, and in some squamates (Table S1). We measure a very slight albeit non-significant phylogenetic signal for both the cervical and the caudal locus $(\lambda=0.471, p=0.189)$. This slight phylogenetic signal is probably driven by clades displaying homogenous loci position such as Marsupialia, Glires (rodents + lagomorphs), and Archosauria (Fig. S5).

Despite homogeneity within some clades, we could not reconstruct any of the ancestral 193 locus is reconstructed as ancestral in Marsupialia and Glires (Fig. 3C), and as the most 194 likely ancestral state in some subclades of Squamata (Fig. S5). The ancestral condition is 195 unresolved in Squamata and its subclades. In all other clades, including Amniota, we find 196 that the cervical locus is the probable ancestral condition (Fig. 3C).

\section{Neurocentral fusion}

Our observations on the neurocentral fusion of Stereosternum are based on specimen 
201 are unfused in dorsal vertebrae, whereas the posteriormost caudals are completely fused

202 (Fig. 2C). This suggests a caudocranial (from tail to head) direction of neurocentral fusion, 203 probably from a caudal locus.

204 In stark contrast to the three other ossification patterns, a cervical locus is neither the most common nor the reconstructed ancestral condition for neurocentral fusion in amniotes. Instead, neurocentral fusion ancestrally started from a caudal locus with fusion proceeding anteriorly (Fig. 3D). The caudal locus is maintained in most mammals and non-avian reptiles, but is absent in birds, rodents and lagomorphs, humans, as well as in a few squamates and dinosaurs (Table S1).

210 Birds deviate from the ancestral condition by adopting an anteroposterior neurocentral 211 fusion starting from a cervical locus (Fig. 3D). The transition from a caudal to a cervical 212 locus already occurred prior to Aves among non-avian dinosaurs: the ornithomimosaurian 213 Nqwebasaurus and the tyrannosaurid Dilong also possess a cervical locus (Table S1), 214 suggesting that this locus appeared in Coelurosauria (Fig. S6, S7). Moreover, both the 215 cervical and the caudal locus are present in Dilong, showing that both loci coexisted in 216 early coelurosaurians until the caudal locus later disappeared in more derived avian 217 clades.

218 In addition to coelurosaurs, several mammals and dinosaurs also acquired a cervical 219 locus in addition to the caudal one (Table S1). Moreover, certain squamates possess only 220 the cervical locus and have lost the caudal locus. This suggests that the presence of a cervical locus is a rather plastic trait that emerged independently multiple times.

Among mammals, rodents and lagomorphs adopt a sacral locus, probably through the anterior shifting of the caudal locus (Fig. 3D). Humans are the only amniotes to present both a thoracic and a lumbar locus. It is possible that the thoracic and the lumbar locus respectively result from the posterior shifting of a cervical locus and the anterior shifting of a caudal one. Additional data on primates would be necessary for this hypothesis to be 227 tested.

\section{Discussion}

Axial ossification patterns and Hox genes

231 Despite their evolutionary history spanning over 300 million years and their great 232 diversity and disparity, amniotes are surprisingly conservative with respect to axial 233 ossification patterns. This is also reflected in the significant phylogenetic signal recovered 234 in each of the patterns (Fig. S1-S7). It is remarkable that in the vast majority of amniotes 
235 some loci as well as the directionality of axial ossification has been maintained since the 236 initial evolution of Amniota (Fig. 3). This is particularly interesting considering the disparity 237 of vertebral morphologies and functions in the clade, and the diverse patterns and modes 238 of axial regionalization that have been recognized (Böhmer et al., 2018; Bui and Larsson, 239 2021; Jones et al., 2018; Krings et al., 2014; Terray et al., 2020).

240 It seems likely that the observed conservatism of axial ossification patterns is due to 241 constraints imposed by the underlying genetic processes during vertebral development.

242 While genetic material does not preserve in deep geological time, comparisons with 243 modern representatives can provide some insights into the genetic underpinnings of axial 244 patterning in fossil taxa (Böhmer et al., 2015; Head and Polly, 2015; Müller et al., 2010). 245 In this context, the role of Hox genes and their respective regulators in directing axial 246 patterning in vertebrates specifically and animals more broadly, has long been known 247 (Böhmer et al., 2015; Head and Polly, 2007; McGinnis and Krumlauf, 1992; Shubin et al., 248 2009; Wellik, 2007).

249 Moreover, a number of studies have shown that overlapping expression domains of 250 Hox genes along the anteroposterior axis of the embryo directly impact vertebral 251 morphologies in the adult (Böhmer, 2017; Böhmer et al., 2015; Burke et al., 1995; Galis, 252 1999; Hautier et al., 2014, 2010; Ohya et al., 2005) and that the ranges of Hox gene 253 expression domains can also influence the relative timing of ossification in vertebral 254 elements (Bui and Larsson, 2021; Hautier et al., 2014, 2010). Therein, certain Hox4-10 255 paralogues linked to vertebral regionalization have been suggested to have been retained 256 in Amniota since the last common ancestor of the clade (Head and Polly, 2015). Therefore, 257 axial ossification models including those of fossil taxa may indeed reflect to some degree 258 how developmental constraints prevented or channelized evolutionary innovation. 259 However, the more detailed roles that Hox and other genes play in directing ossification 260 and fusion patterns in extinct and extant taxa alike remains to be investigated.

261 Among the main clades of amniotes, two stand out in terms of these patterns: 262 mammals notably diverge from the ancestral amniote condition for centrum and neural 263 arch ossification, and to a lesser extent, birds also diverge from the ancestral amniotic 264 neurocentral fusion pattern. In addition, several subclades within mammals and birds 265 diverge from the ancestral condition of their parent taxa: Paleognathae and Talpidae for 266 centrum ossification, Australidelphia for neural arch ossification, Marsupialia for neural 267 arch fusion, Glires for neural arch fusion and neurocentral fusion (Fig. 3). This suggests 
268 that mammals and birds have a greater potential for variation in their axial ossification 269 patterns than the other main groups of amniotes, like squamates and turtles.

270 Mammals and birds have a strongly regionalized vertebral column, which means that 271 their column is segmented into more morphologically and functionally distinct regions than 272 other amniotes (Böhmer et al., 2018; Bui and Larsson, 2021; Jones et al., 2020, 2018; 273 Krings et al., 2014; Terray et al., 2020). Strongly regionalized columns result from complex 274 interactions between Hox genes (Burke et al., 1995; Young et al., 2009), and changes in 275 Hox gene expression domains can in turn influence the timing of ossification (Bui and 276 Larsson, 2021; Hautier et al., 2014, 2010). With their strongly regionalized columns, 277 mammals and birds possess a more strongly modularized vertebral column, which in turn 278 may provide a greater potential for the accumulation of changes within each module and 279 in the overall patterning and timing of ossification (Jones et al., 2019). Therefore, the 280 greater diversity of axial ossification patterns in mammals and birds may directly results 281 from their regionalization.

282 Although the current state of knowledge prevents us from statistically testing the links 283 between regionalization and axial ossification, there are a few cases where changes in 284 regionalization parallel modifications of axial ossification patterns. For instance, the 285 transition in coelurosaurian dinosaurs from a caudal to a cervical locus of neurocentral 286 fusion strikingly mirrors the progressive loss of articulation in tail vertebrae in favor of a 287 fused pygostyle in birds, a loss probably caused itself by variations in Hox genes (Rashid 288 et al., 2014). Similarly, the adoption of a fixed number of seven cervicals in mammals 289 through Hox gene mutations coincides with the acquisition of an additional thoracic locus 290 of neural arch ossification (Böhmer, 2017; Müller et al., 2010).

291 While these examples are consistent with our hypothesis and the study of vertebral 292 regionalization has expanded rapidly in recent years (Bui and Larsson, 2021; Jones et al., 293 2020, 2019; Terray et al., 2020), some amniote clades remain under-represented in terms 294 of quantitative data on the topic. For instance, our hypothesis predicts that non-avian 295 reptiles should have less complex patterns of Hox gene expression during axial 296 development matching the poorly regionalized vertebral column in the adults. Determining 297 whether this is indeed the case would provide more insights into potential links between 298 these two processes. This shows how investigating vertebral regionalization and 299 development in further non-model amniote taxa would be crucial to understand the 300 evolutionary history of the vertebral column in amniotes. 


\section{Scenarios for the evolution of axial ossification patterns}

We propose the following ancestral condition for axial ossification patterns in amniotes: (i) pleurocentrum ossification proceeding posteriorly from two loci in the cervical and the thoracic region, (ii) neural arch ossification proceeding posteriorly from a single cervical locus, (iii) neural arch fusion progressing posteriorly from a single cervical locus in a "zipper-like" pattern, and (iv) neurocentral fusion proceeding anteriorly from a caudal locus. From this ancestral condition, their exact evolutionary development remains obscure due to the current lack of fossil data. On the reptilian branch of Amniota, the exceptional specimens of Stereosternum provide unique data from the fossil record that fill the gap that data based on extant animals alone leave behind. For each axial ossification pattern, Stereosternum displays the reconstructed ancestral reptilian condition, and for all patterns but centrum ossification, it even displays the ancestral amniote condition. The observations in this ancient amniote taxon therefore lend some support to our reconstruction.

On the mammalian branch however, the lack of information for vertebral ossification patterns in non-mammalian synapsids is problematic. Axial ossification patterns have never been documented in any pelycosaur-grade synapsid in therapsids, in Mesozoic or even Cenozoic mammals. Additional data from fossil synapsids would greatly help to refine the resolution and robustness of the reconstructed evolutionary history of these traits in amniotes.

Based on the current data and depending on what will eventually be found in synapsids in the future, we predict three scenarios: (i) fossil synapsids show patterns of axial ossification resembling those of reptiles. In that case, the observed reptilian patterns would reflect the ancestral condition in all amniotes. The condition observed in modern mammals would then constitute a synapomorphy adopted relatively late in their evolutionary history. This would support our speculation that the specific axial ossification patterns found in crown-mammals may be connected to the evolution of a stronger vertebral regionalization in therapsids (see also Jones et al., 2020); (ii) fossil synapsids show patterns of axial ossification resembling those of crown-mammals. This would imply that the two main branches of Amniota each developed their own specific modes of axial ossification very early in their evolutionary history, potentially at the time of their original dichotomy. This would in return suggest that axial ossification patterns might have played a role in this dichotomy; (iii) fossil synapsids show unique patterns of axial ossification. This scenario would combine the other two, with a dichotomy in axial ossification patterns at the base of 
336 Amniota and the innovation of another, different condition in crown-mammals. Regardless

337 of which scenario might be supported once additional data on fossil synapsids becomes

338 available, it will yield important implications for the evolutionary history of morphological

339 diversity in amniotes.

341 Conclusions

342 Reviewing the literature and with additional data from exceptionally well-preserved fossils,

343 we reconstruct the ancestral axial ossification and fusion patterns in amniotes:

344 - Centra ossify from neck to tail, starting from two loci in the cervical and the thoracic

345 region.

346 - Neural arches also ossify posteriorly but from a single cervical locus.

347 - Neural arches fuse together in a "zipper-like" pattern, starting in a cervical locus and 348 progressing posteriorly.

349 - Neurocentral fusion begins in the caudal region and progresses anteriorly towards the 350 head.

351 Despite the long evolutionary history of amniotes, all four axial ossification patterns 352 show a strong phylogenetic signal and appear to have been quite stable over time. We 353 propose that this conservatism may be linked to constraints posed by underlying 354 developmental processes across amniotes. Our study also highlights variability in the axial 355 ossification patterns of birds and mammals. We suggest a correlation between this 356 variability and the strong vertebral regionalization existing in these clades. This study 357 provides a framework for further research on axial ossification in fossil and modern 358 amniotes, but more fossil and molecular data will be necessary to further resolve the 359 ancestral ossification patterns within Amniota.

\section{Materials and Methods}

362 Our study focuses on four major patterns of axial ossification: centrum ossification, 363 neural arch ossification, neural arch fusion, and neurocentral fusion. For each of these 364 patterns, we reviewed the existing literature on extant and fossil amniotes and gathered 365 information on two key aspects: the number of loci in the spine from where the patterns 366 start and the position of these loci in the vertebral column. Very little information was 367 available on these patterns in the fossil record. The axial skeleton of juvenile specimens 368 of extinct amniotes has rarely been described in sufficient detail for the scope of our study. 
369 Fortunately, based on exceptional specimens of the early Permian mesosaurid reptile 370 Stereosternum tumidum, we were able document patterns of axial ossification in one of 371 the most basal clades of amniotes. In addition, to complete missing information on the 372 patterns of some extant taxa, observations made on specimens from the collections of the 373 Museum für Naturkunde in Berlin, Germany were included (Table S1).

A composite tree of all studied species was constructed in Mesquite 3.6(Maddison and Maddison, 2019) combining recently published phylogenies (Fig. S8). In addition to the taxa for which vertebral ossification data was available, fossil taxa were included in the tree to obtain a more accurate time-calibration following recommendations from the Fossil

378 Calibration Database (Ksepka et al., 2015). The resulting tree was time-calibrated based on occurrence dates from the Paleobiology Database (Uhen and Sessa, 2013) and using 380 equal time-scaling method. A subset of the time-calibrated tree was then generated for each of the four ossification patterns by trimming the original tree to match the taxon sample with available data.

In each studied taxon, the four ossification patterns were characterized in terms of number of loci and the position of these loci in the vertebral column. The presence or absence of a locus was noted in five sections of the vertebral column for each pattern: (i) cervical, (ii) upper dorsal/thoracic, (iii) lower dorsal/lumbar (iv) sacral, and (v) caudal. The presence/absence of a locus in these sections was scored as a binary character. As a result, each ossification pattern was decomposed into a matrix of four binary characters, one for each section of the vertebral column (Table S1). The binary characters were then tested for phylogenetic signal using Pagel's $\lambda$ (Pagel, 1999). Ancestral state reconstruction was performed on each character twice, once using maximum likelihood with symmetrical rates of evolution, and once using maximum parsimony with sequential transition costs.

Tree calibration and trimming, statistical analyses, and ancestral character reconstructions were performed using the ape (Paradis and Schliep, 2019), castor (Louca

395 and Doebeli, 2017), paleotree (Bapst, 2012), and phytools (Revell, 2012) packages in $R$

396 v.4.0.5 (R. Core Team, 2021). Code in supplements (Dataset S1).

\section{Acknowledgments}

399 We thank R. Brocke (Senckenberg Naturmuseum, Frankfurt), A. Carvalho (Museu de 400 Zoologia da Universidade de São Paulo, SP), P. Eckhoff (Museum für Naturkunde, Berlin), 401 C. Funk (Museum für Naturkunde, Berlin), O. Rauhut (Bayerische Staatssammlung für 402 Paläontologie und Geologie, Munich), M.-O. Rödel (Museum für Naturkunde, Berlin) for 
403 access to collections and specimen loans. Very special thanks to A. Carvalho and $\mathrm{H}$.

404 Zaher (MZSP) who helped us obtain crucial photographs of MZSP despite the pandemic.

405 Additional thanks to J. Müller, M.J MacDougall and Y. Haridy (Museum für Naturkunde,

406 Berlin) for discussion and their helpful comments during the drafting of this article. This

407 work was supported by the German Research Foundation (DFG FR 2457/6-1).

References

Bapst DW. 2012. paleotree : an R package for paleontological and phylogenetic analyses of evolution: Analyses of Paleo-Trees in R. Methods Ecol Evol 3:803-807. doi:10.1111/j.2041-210X.2012.00223.x

Böhmer C. 2017. Correlation between Hox code and vertebral morphology in the mouse: towards a universal model for Synapsida. Zool Lett 3:8. doi:10.1186/s40851-0170069-4

Böhmer C, Amson E, Arnold P, van Heteren AH, Nyakatura JA. 2018. Homeotic transformations reflect departure from the mammalian 'rule of seven' cervical vertebrae in sloths: inferences on the Hox code and morphological modularity of the mammalian neck. BMC Evol Biol 18:84. doi:10.1186/s12862-018-1202-5

Böhmer C, Rauhut OWM, Wörheide G. 2015. Correlation between Hox code and vertebral morphology in archosaurs. Proc $R$ Soc $B$ Biol Sci 282:20150077. doi:10.1098/rspb.2015.0077

Bui H-NN, Larsson HCE. 2021. Development and evolution of regionalization within the avian axial column. Zool J Linn Soc 191:302-321. doi:10.1093/zoolinnean/zlaa038

Burke AC, Nelson CE, Morgan BA, Tabin C. 1995. Hox genes and the evolution of vertebrate axial morphology. Development 121:333-346.

Clack JA. 2012. Gaining Ground: The Origin and Evolution of Tetrapods, 2nd ed. Indiana University Press.

Danto M, Witzmann F, Pierce SE, Fröbisch NB. 2017. Intercentrum versus pleurocentrum growth in early tetrapods: A paleohistological approach. $J$ Morphol. doi:10.1002/jmor.20709

Fleming A, Kishida MG, Kimmel CB, Keynes RJ. 2015. Building the backbone: the development and evolution of vertebral patterning. Development 142:1733-1744. doi:10.1242/dev.118950

Gadow H. 1896. On the evolution of the vertebral column of Amphibia and Amniota. Philos Trans $R$ Soc Lond Ser $B$ Contain Pap Biol Character 187:1-57. doi:10.1098/rstb. 1896.0001

Galis F. 1999. Why do almost all mammals have seven cervical vertebrae? Developmental constraints, Hox genes, and cancer. $J$ Exp Zool 285:19-26. doi:10.1002/(SICI)1097-010X(19990415)285:1<19::AID-JEZ3>3.0.CO;2-Z

Hautier L, Charles C, Asher RJ, Gaunt SJ. 2014. Ossification sequence and genetic patterning in the mouse axial skeleton. J Exp Zoolog B Mol Dev Evol 322:631642. doi:10.1002/jez.b.22590

Hautier L, Weisbecker V, Goswami A, Knight F, Kardjilov N, Asher RJ. 2011. Skeletal ossification and sequence heterochrony in xenarthran evolution. Evol Dev 13:460476. doi:10.1111/j.1525-142X.2011.00503.x

Hautier L, Weisbecker V, Sánchez-Villagra MR, Goswami A, Asher RJ. 2010. Skeletal development in sloths and the evolution of mammalian vertebral patterning. Proc Natl Acad Sci 107:18903-18908. doi:10/cvbj2s 
450 Head JJ, Polly PD. 2015. Evolution of the snake body form reveals homoplasy in amniote Hox gene function. Nature 520:86.

Head JJ, Polly PD. 2007. Dissociation of somatic growth from segmentation drives gigantism in snakes. Biol Lett 3:296-298. doi:10.1098/rsbl.2007.0069

Irmis RB. 2007. Axial skeleton ontogeny in the Parasuchia (Archosauria: Pseudosuchia) and its implications for ontogenetic determination in archosaurs. J Vertebr Paleontol 27:350-361. doi:10.1671/0272-4634(2007)27[350:ASOITP]2.0.CO;2

Jones KE, Angielczyk KD, Pierce SE. 2019. Stepwise shifts underlie evolutionary trends in morphological complexity of the mammalian vertebral column. Nat Commun 10:5071. doi:10.1038/s41467-019-13026-3

Jones KE, Angielczyk KD, Polly PD, Head JJ, Fernandez V, Lungmus JK, Tulga S, Pierce SE. 2018. Fossils reveal the complex evolutionary history of the mammalian regionalized spine. Science 361:1249-1252. doi:10.1126/science.aar3126

Jones KE, Gonzalez S, Angielczyk KD, Pierce SE. 2020. Regionalization of the axial skeleton predates functional adaptation in the forerunners of mammals. Nat Ecol Evol 4:470-478. doi:10.1038/s41559-020-1094-9

Kölliker A. 1876. Entwicklungsgeschichte des Menschen und der höheren Thiere. W. Engelmann.

Krings M, Nyakatura JA, Fischer MS, Wagner H. 2014. The cervical spine of the American barn owl (Tyto furcata pratincola): I. Anatomy of the vertebrae and regionalization in their S-shaped arrangement. PLOS ONE 9:e91653. doi:10.1371/journal.pone.0091653

Ksepka DT, Parham JF, Allman JF, Benton MJ, Carrano MT, Cranston KA, Donoghue PCJ, Head JJ, Hermsen EJ, Irmis RB, Joyce WG, Kohli M, Lamm KD, Leehr D, Patané JL, Polly PD, Phillips MJ, Smith NA, Smith ND, Van Tuinen M, Ware JL, Warnock RCM. 2015. The fossil calibration database-a new resource for divergence dating. Syst Biol 64:853-859. doi:10.1093/sysbio/syv025

Lawson LY, Harfe BD. 2017. Developmental mechanisms of intervertebral disc and vertebral column formation. WIREs Dev Biol 6:e283. doi:10.1002/wdev.283

Louca S, Doebeli M. 2017. Efficient comparative phylogenetics on large trees. Bioinformatics 34:1053-1055. doi:10.1093/bioinformatics/btx701

Maddison WP, Maddison DC. 2019. Mesquite: a modular system for evolutionary analysis.

Maxwell EE, Larsson HCE. 2009. Comparative ossification sequence and skeletal development of the postcranium of palaeognathous birds (Aves: Palaeognathae). Zool J Linn Soc 157:169-196. doi:10.1111/j.1096-3642.2009.00533.x

McGinnis W, Krumlauf R. 1992. Homeobox genes and axial patterning. Cell 68:283-302. doi:10.1016/0092-8674(92)90471-N

Müller J, Scheyer TM, Head JJ, Barrett PM, Werneburg I, Ericson PGP, Pol D, SánchezVillagra MR. 2010. Homeotic effects, somitogenesis and the evolution of vertebral numbers in recent and fossil amniotes. Proc Natl Acad Sci 107:2118-2123. doi:10.1073/pnas.0912622107

Ohya YK, Kuraku S, Kuratani S. 2005. Hox code in embryos of Chinese soft-shelled turtle Pelodiscus sinensis correlates with the evolutionary innovation in the turtle. J Exp Zoolog B Mol Dev Evol 304B:107-118. doi:https://doi.org/10.1002/jez.b.21027

Pagel M. 1999. Inferring the historical patterns of biological evolution. Nature 401:877884. doi: $10.1038 / 44766$

Paradis E, Schliep K. 2019. ape 5.0: an environment for modern phylogenetics and evolutionary analyses in R. Bioinformatics 35:526-528. doi:10.1093/bioinformatics/bty633 
499 Prochel J. 2006. Early skeletal development in Talpa europaea, the common European $500 \quad$ mole. Zoolog Sci 23:427-434.

501 R. Core Team. 2021. R: a language and environment for statistical computing. Vienna, Austria: R Foundation for Statistical Computing.

Rashid DJ, Chapman SC, Larsson HC, Organ CL, Bebin A-G, Merzdorf CS, Bradley R, Horner JR. 2014. From dinosaurs to birds: a tail of evolution. EvoDevo 5:25. doi:10.1186/2041-9139-5-25

Revell LJ. 2012. phytools: an R package for phylogenetic comparative biology (and other things): phytools: R package. Methods Ecol Evol 3:217-223. doi:10.1111/j.2041210X.2011.00169.x

Shubin N, Tabin C, Carroll S. 2009. Deep homology and the origins of evolutionary novelty. Nature 457:818-823. doi:10.1038/nature07891

Terray L, Plateau O, Abourachid A, Böhmer C, Delapré A, de la Bernardie X, Cornette R. 2020. Modularity of the neck in birds (Aves). Evol Biol 47:97-110. doi:10.1007/s11692-020-09495-w

Uhen MD, Sessa J. 2013. The Paleobiology Database. paleobiodb.org. http://paleobiodb.org/\#/

Wellik DM. 2007. Hox patterning of the vertebrate axial skeleton. Dev Dyn 236:24542463. doi:10.1002/dvdy.21286

Young T, Rowland JE, van de Ven C, Bialecka M, Novoa A, Carapuco M, van Nes J, de Graaff W, Duluc I, Freund J-N, Beck F, Mallo M, Deschamps J. 2009. Cdx and Hox genes differentially regulate posterior axial growth in mammalian embryos. Dev Cell 17:516-526. doi:10.1016/j.devcel.2009.08.010

Zhang G. 2009. An evo-devo view on the origin of the backbone: evolutionary development of the vertebrae. Integr Comp Biol 49:178-186. doi:10.1093/icb/icp061 
bioRxiv preprint doi: https://doi.org/10.1101/2021.09.23.461462; this version posted September 24, 2021. The copyright holder for this preprint (which was not certified by peer review) is the author/funder, who has granted bioRxiv a license to display the preprint in perpetuity. It is made available under aCC-BY-NC-ND 4.0 International license.

\section{Figures}

527

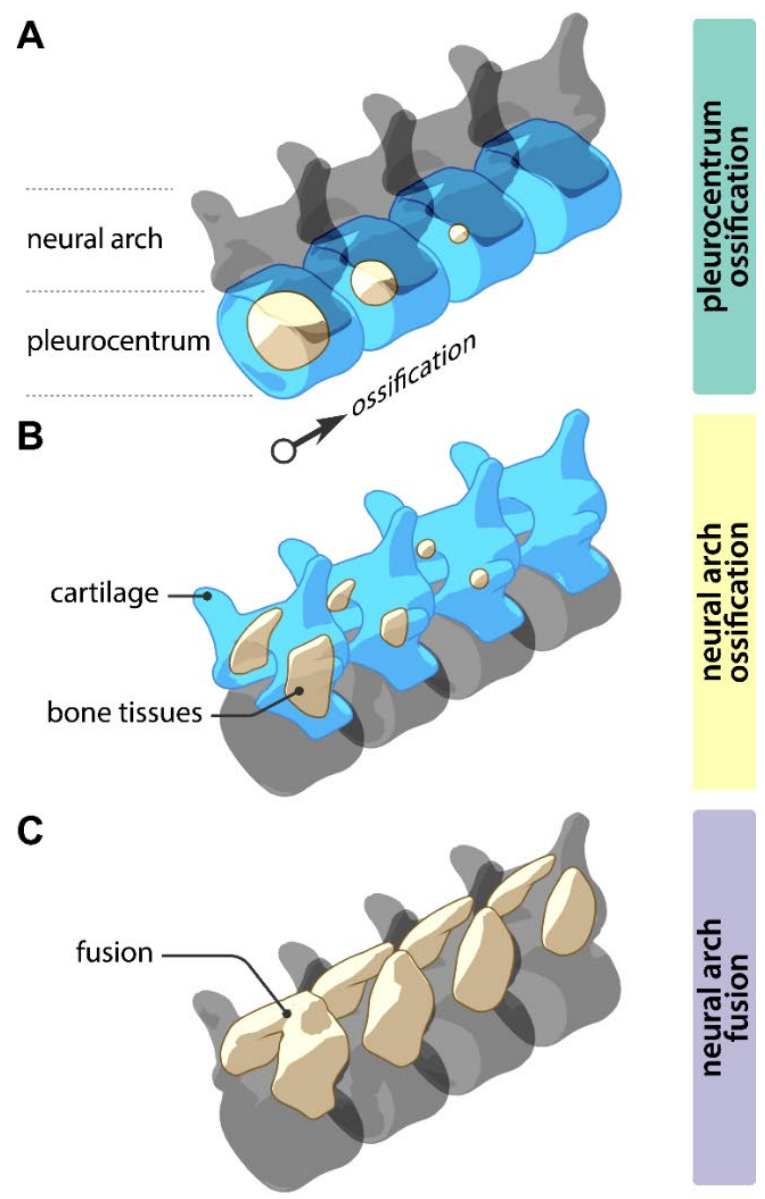

D

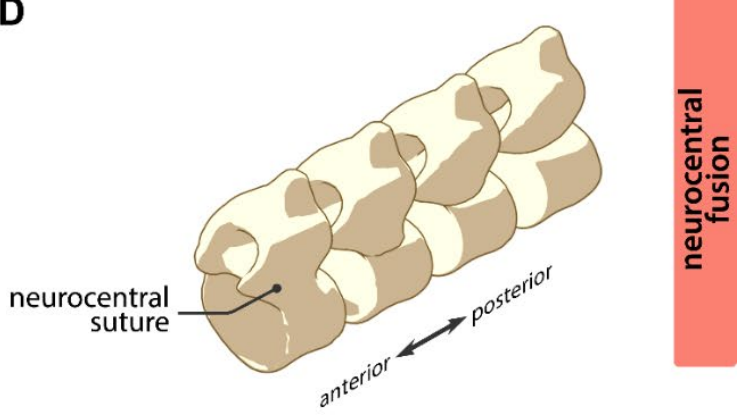

529 Figure 1. Schematic illustration of the four vertebral ossification patterns examined in the 530 present study. A. pleurocentrum ossification. B. neural arch ossification. C. neural arch 531 fusion. D. neurocentral fusion. 
A

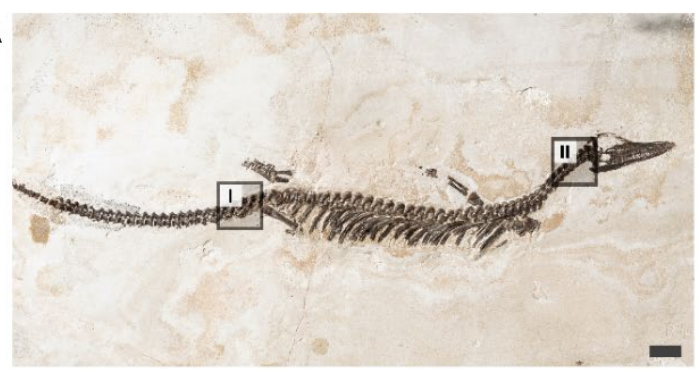

B

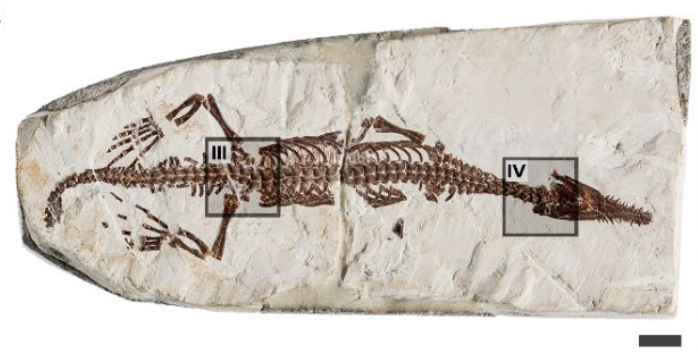

C

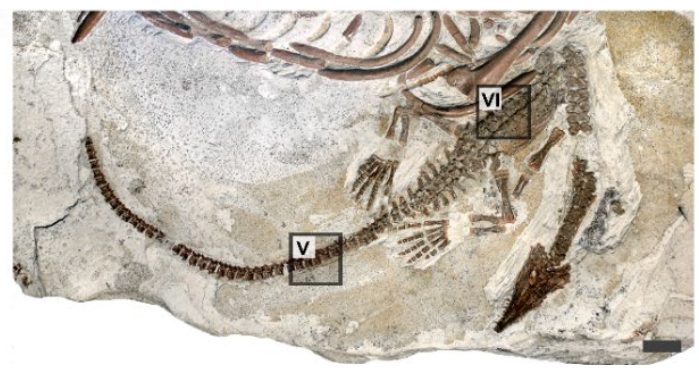

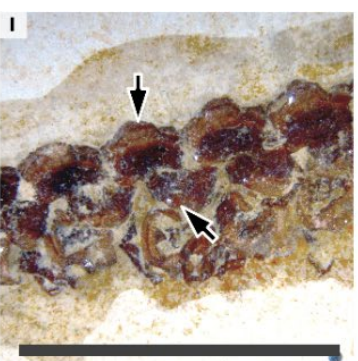
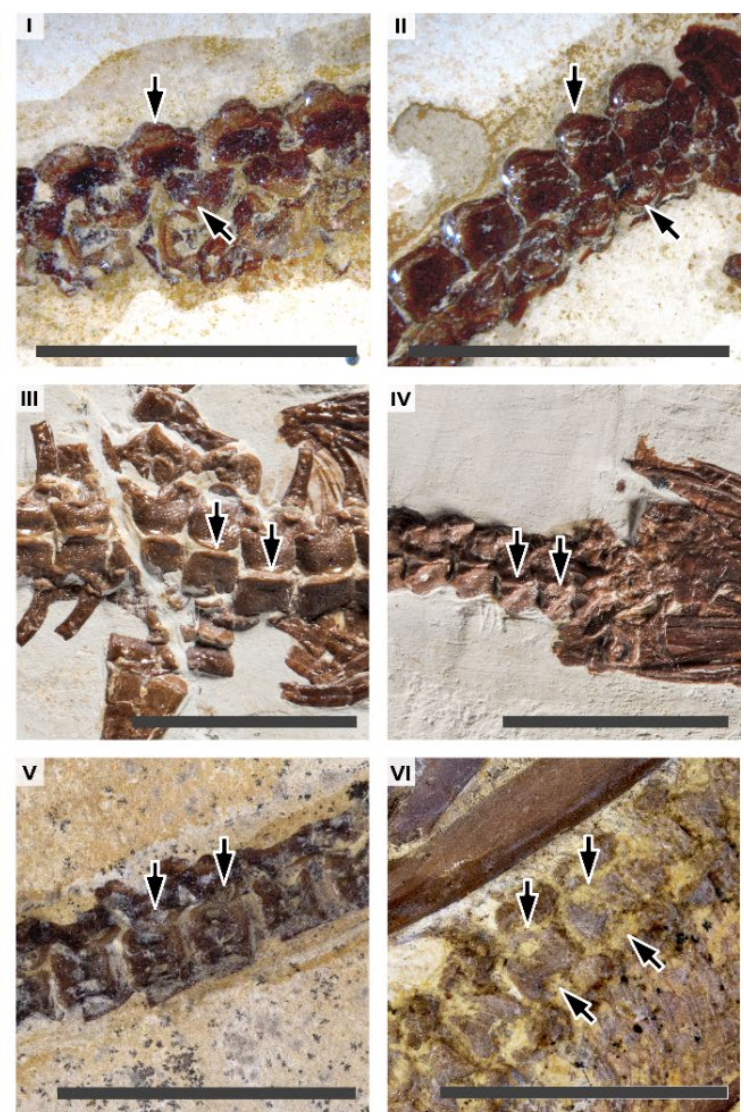

533 Figure 2. Juvenile specimens of Stereosternum tumidum showing axial ossification patterns. A. SMF-R-4512, showing gradients of pleurocentrum and neural arch ossification; B. BSPG 1979 I 37, showing a gradient of neural arch fusion; C. MZSP-PV 1301 , showing a gradient of neurocentral fusion. Specimens are oriented with the posterior region to the left and the anterior region to the right. Dark grey squares highlight the areas

539

540 shown in close-up with the matching numbering. Arrows highlight differences in degree of ossification or fusion between two adjacent close-ups. Scale bars: $10 \mathrm{~mm}$. 

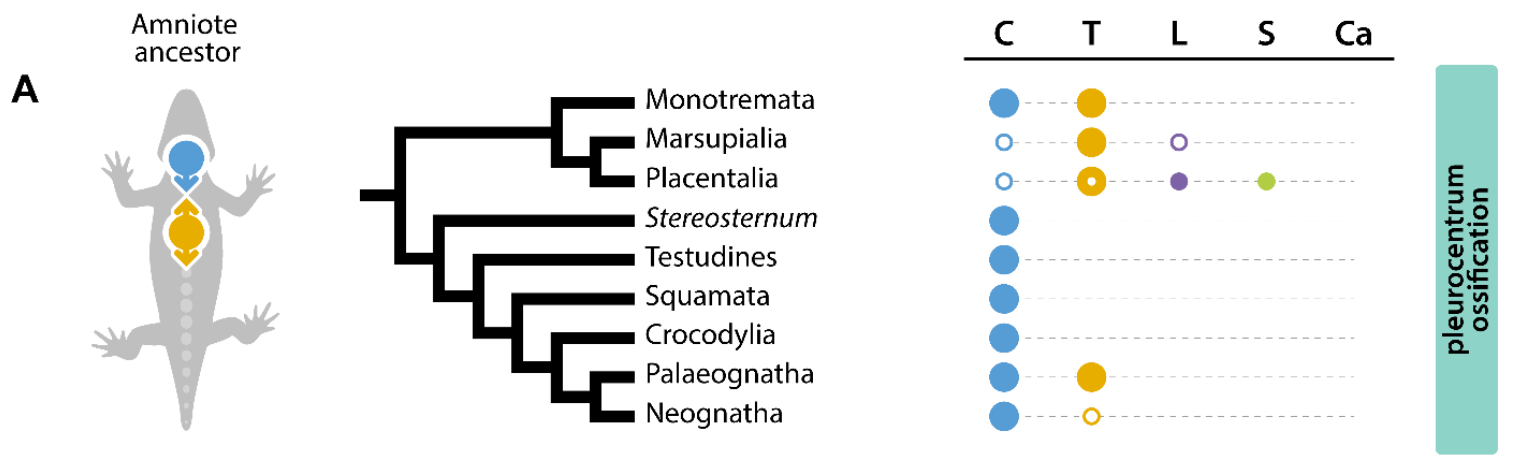

B

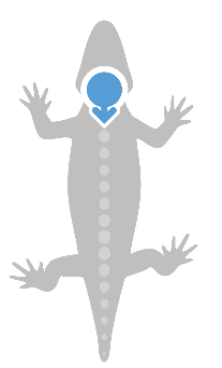

C

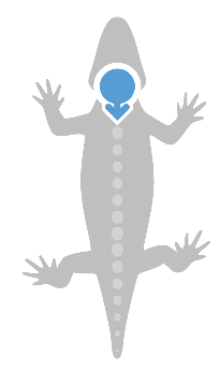

D

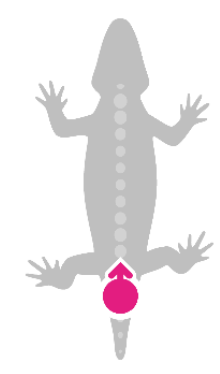

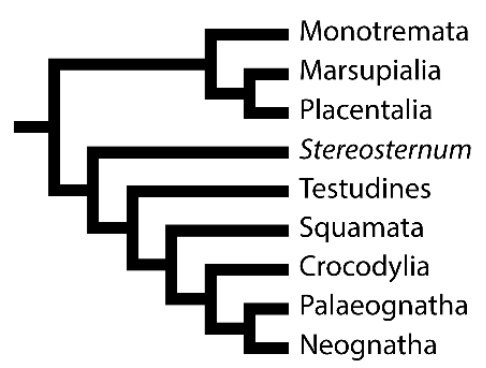
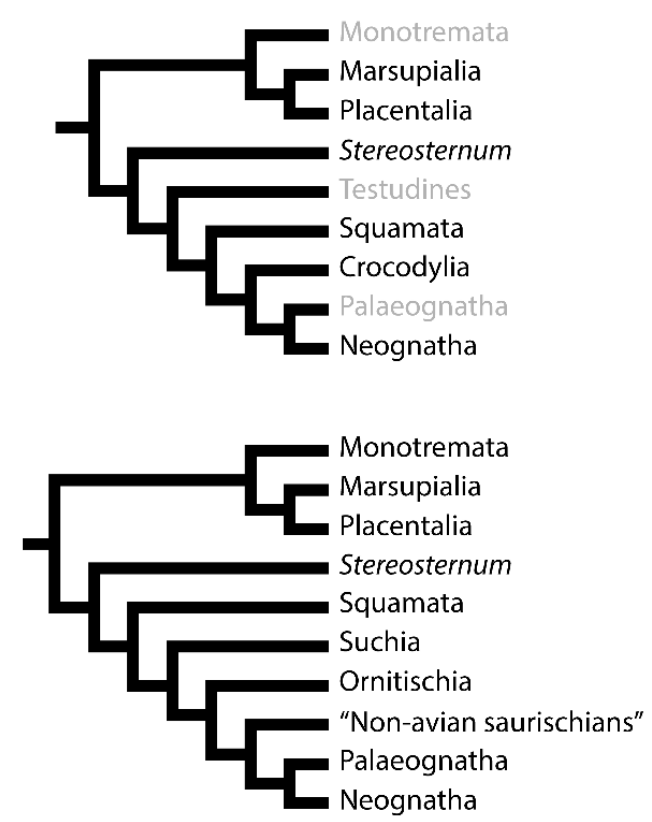
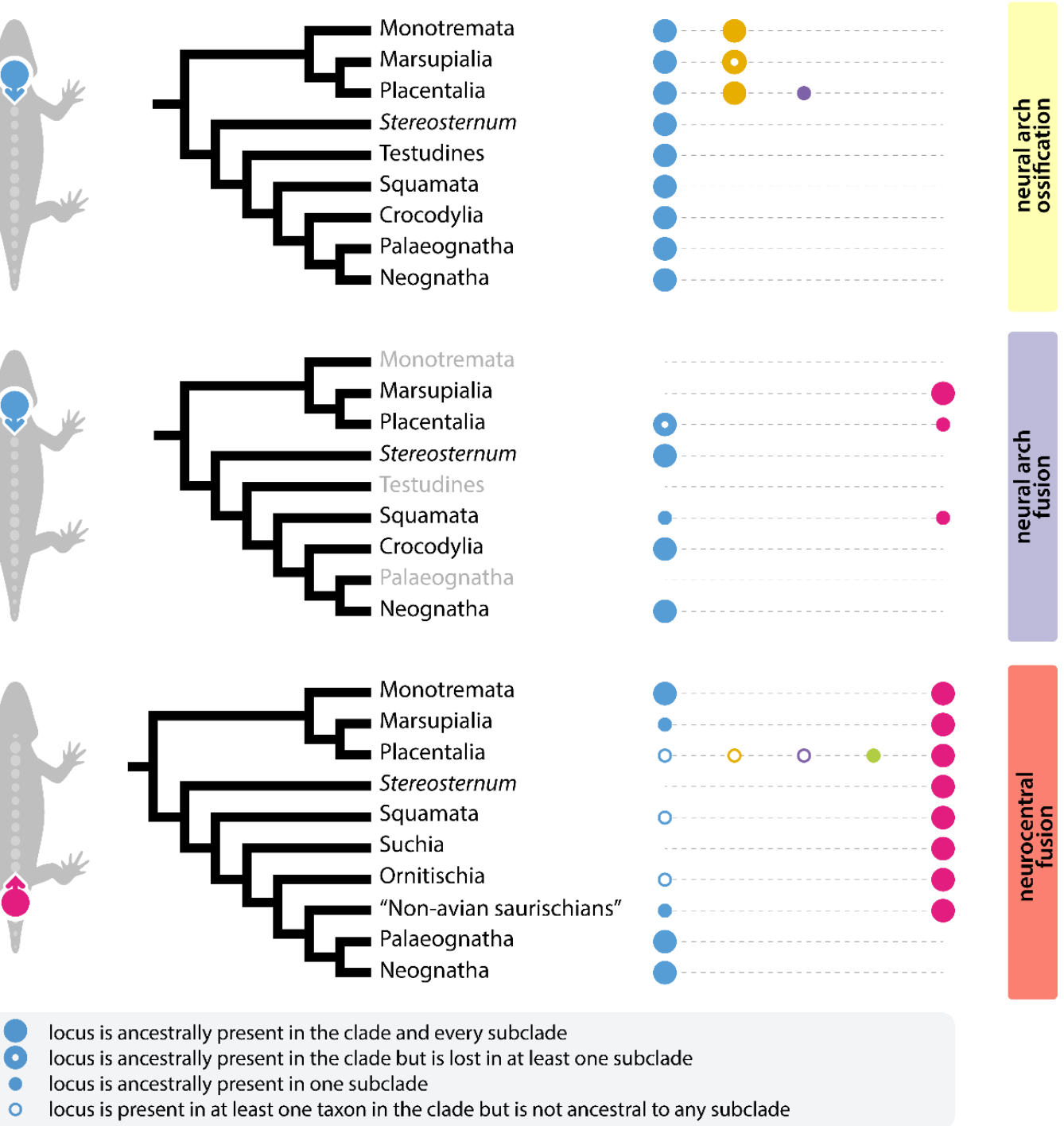

Figure 3. Distribution and ancestral state reconstruction of loci of axial ossification and fusion in amniotes: A. centrum ossification; B. neural arch ossification; C. neural arch fusion; D. neurocentral fusion. Abbreviations: C, cervical; T, thoracic/anterior dorsal; L, lumbar/posterior dorsal; S, sacral; $\mathrm{Ca}$, caudal. Colored circles on the grey silhouette represent the reconstructed ancestral condition in amniotes and arrows show the direction of ossification/fusion from each locus. The same color code for loci positions is used all over the figure. Original data in Supplementary figures 1-4. 
neural arch

pleurocentrum

bioRxiv preprint doi: https://doi.org/10.1101/2021.09.23.461462; this version posted September 24, 2021. The copyright holder for

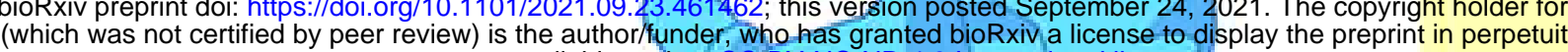
$x^{2}$

C
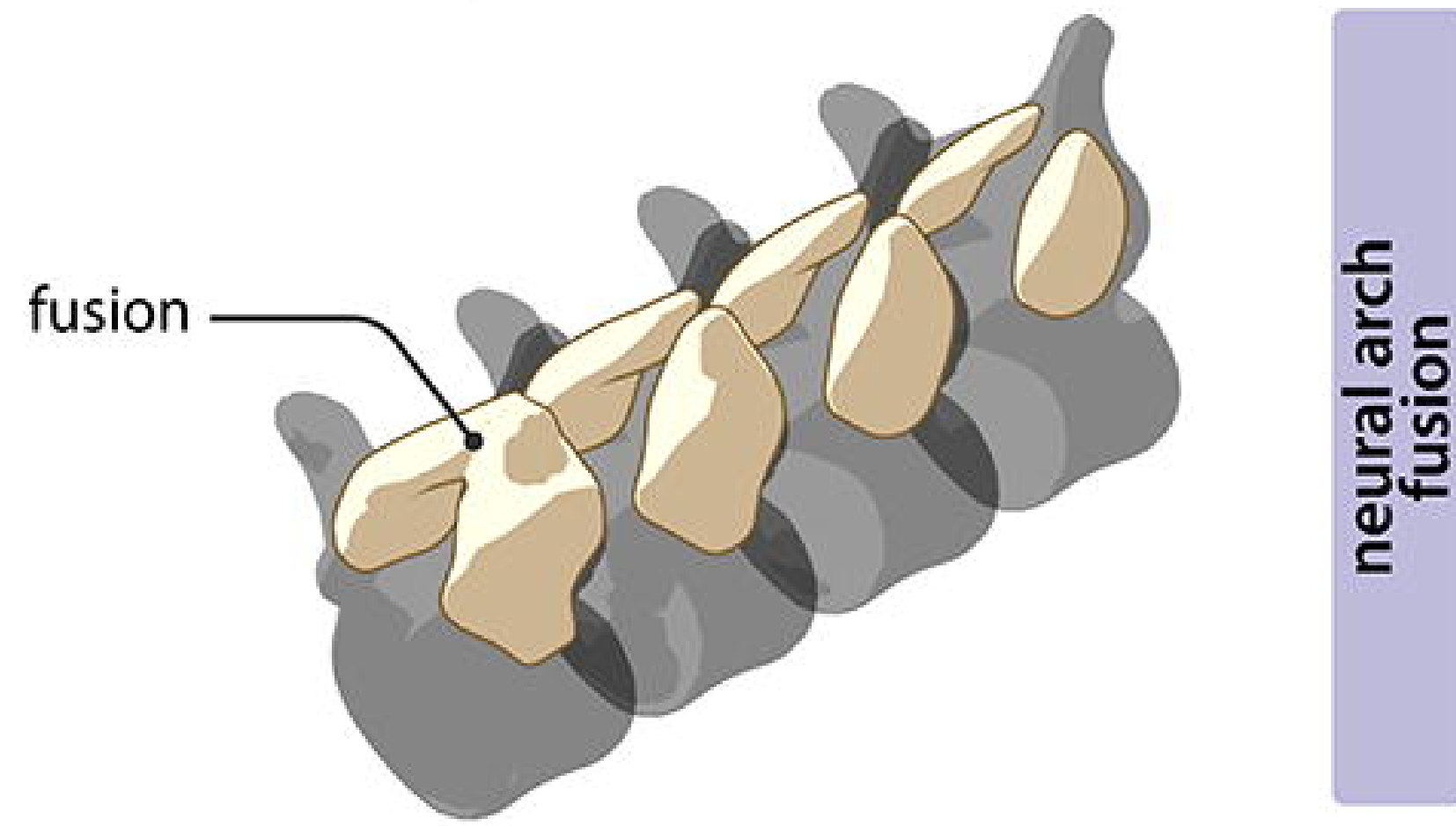

D

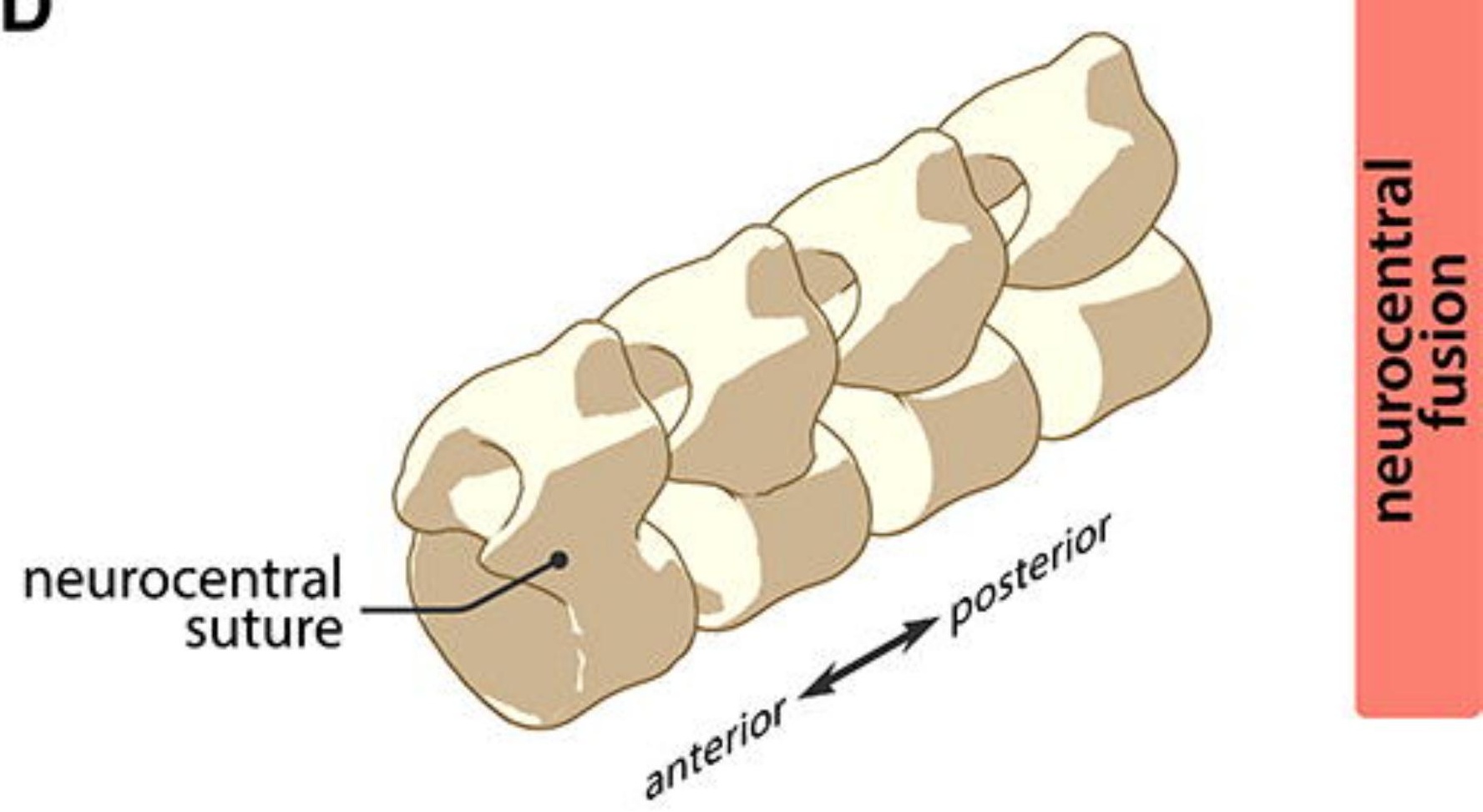




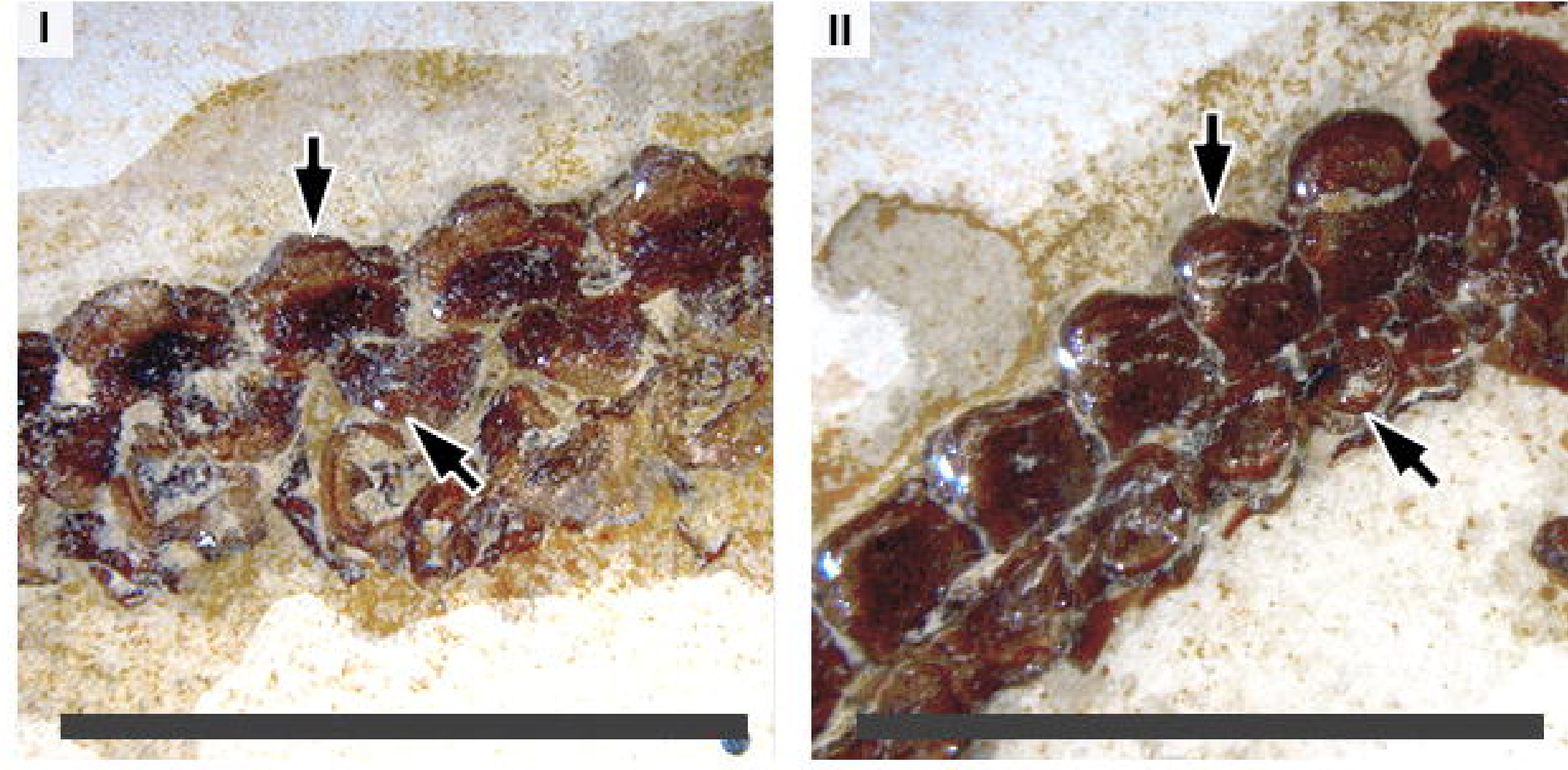

B
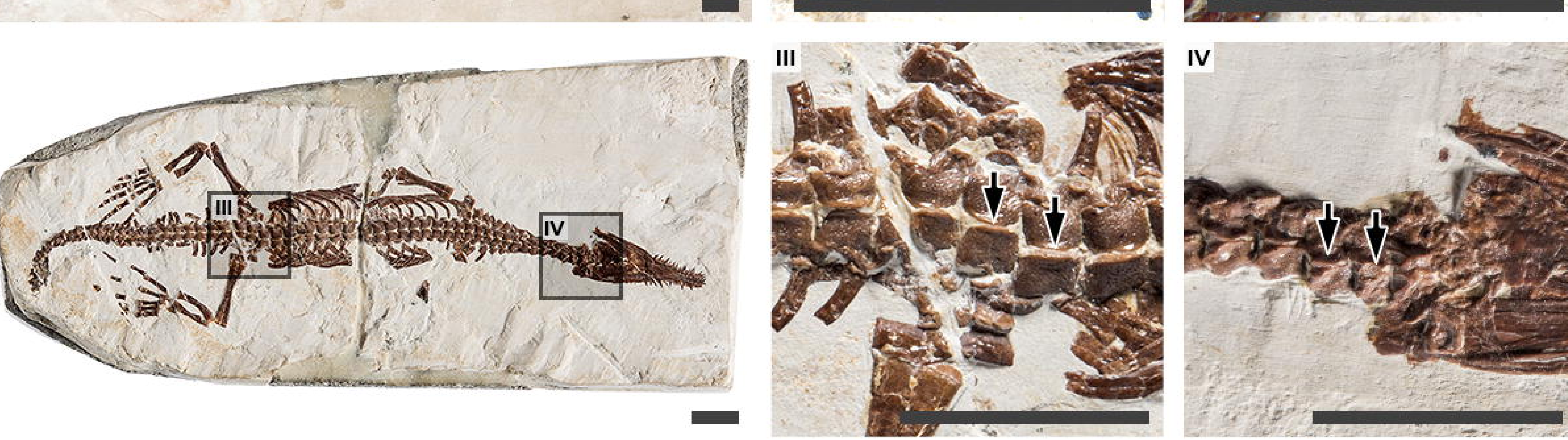

C
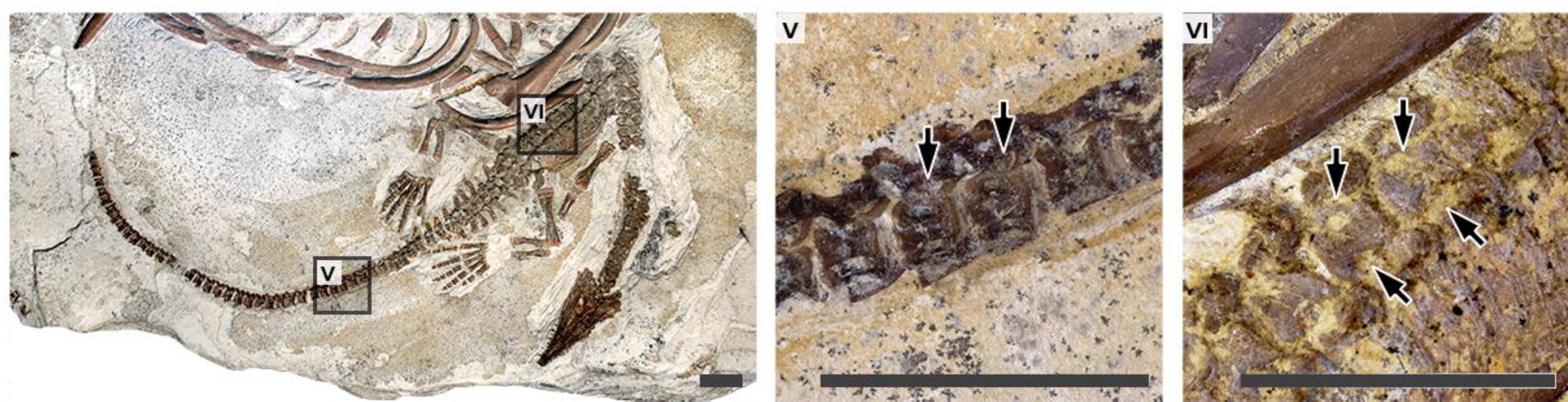
